\title{
Dynamic soaring of UAV within airliner wake vortex in climb regime
}

\author{
Pavel Zikmund, Jiří Matějů \\ Institute of Aerospace Engineering, Brno University of Technology, Czech Republic
}

\begin{abstract}
The paper presents an analysis of a UAV gliding in an airliner wake vortex using dynamic soaring principle. The goal of dynamic soaring is an improvement of flight performance of the UAV following an airliner. The paper extends previously published results of an airliner-UAV climb regime flight formation analysis. Wake vortex model, the UAV basic parameters including drag polar and an airliner climb profile are taken from previous research. Dynamic soaring simulation within wake vortex is performed and evaluated. The presented results provide insight into energy balance and controllability of the UAV flying in an airliner wake vortex.
\end{abstract}

\section{Keywords}

Dynamic soaring, formation flight, wake vortex, self-sustained flight, UAV

\section{Introduction}

Wake vortex is a dangerous phenomenon connected with all heavy airliners. An encounter of another airplane's wake vortex can cause a fatal accident. Let's look at a wake vortex from another point of view. Wake vertices are highenergy flow-fields present in the atmosphere all around the world behind heavy airliners. The paper deals with an extreme flight formation of heavy airliner followed by small unmanned aerial vehicle (UAV) gliding in a wake vortex. The extreme formation has been already studied and the research brought encouraging results in a cruise $^{1,2}$ and even in a climb regime ${ }^{3}$. All these results were obtained for steady flight conditions. This paper extends the research to a dynamic flight with a goal to harvest more energy from a wake vortex compared to a steady flight while ensuring controllability during manoeuvring. The UAV gliding in a wake vortex is called "wake vortex glider" (WVG) below in the text.

Where does the idea about dynamic flight come from? Dynamic soaring (DS) is an interesting principle in flight mechanics which can be used for extraction of energy from a flow-field with velocity gradient. Some birds discovered and use the principle of dynamic soaring when flying above seas and oceans ${ }^{4}$. Boundary layer near water surface contains wind speed gradient. Birds are able to extract enough energy by manoeuvring between flow-fields with different speeds to fly without wing flapping. The same flight pattern performed by an UAV has been in the scope of researchers with a goal to achieve the same benefit as for birds ${ }^{5,6,7}$. DS has been studied for other typical flow-fields featuring wind velocity gradient such as hurricane ${ }^{8}$ and jet stream ${ }^{9}$. Wake vertices contain very strong velocity gradients, giving a promise of successful dynamic soaring application. Further advantage of a wake vortex in DS application is a strongly curved flow-field. Birds need to manoeuvre with 180 degree turns to take advantage of the flat boundary layer. The flowfield in wake vortex is curved, therefore the UAV trajectory is expected to be much smoother compared to a boundary layer DS.

The paper is organized as follows: Section Background of dynamic soaring in wake vortex deals with prerequisites taken from the previous research ${ }^{2,3,10}$. A wake vortex mathematical model, B747 airliner climb profile and WVG drag polar are necessary background for the DS analysis stated in the section.

\section{Corresponding author:}

Pavel Zikmund, Institute of Aerospace

Engineering, Brno University of Technology, Technická 2, 616 69, Brno, Czech Republic.

Email: zikmund@fme.vutbr.cz 
DS principle is also described in the section. Section Methods describes methods used in the research. Equations of motion are laid out at first. Then, an initial trajectory of dynamic soaring in a wake vortex is designed and an optimization method is described. Results of DS trajectory optimization are presented in the next section. The results include kinetic and potential energy gain in DS flight and the trajectory shape for whole climb profile. Required aileron deflection is also evaluated with a goal to validate the extreme formation flight from controllability point of view. Discussion of the results and conclusions are summarized in the two last sections, respectively.

\section{Background of dynamic soaring in wake vortex}

This section recollects results from previous research $^{2,3,10}$. The text is shortened and is included to define initial conditions for the DS analysis. The section deals with a wake vortex model description, B747 airliner climb profile definition and the WVG drag polar estimation.

\section{Wake vortex model description}

Lamb-Oseen wake vortex model was chosen for the DS simulation. It is one of three models used $\mathrm{in}^{3}$. The model is widely used and validated by flight measurement ${ }^{11}$. Induced speeds are defined by following equation (1):

$$
\mathrm{V}_{\mathrm{t}(\mathrm{r})}=\frac{\Gamma_{0}}{2 \pi r}\left[1-\mathrm{e}^{-\alpha\left(\frac{\mathrm{r}}{\mathrm{r}_{\mathrm{c}}}\right)^{2}}\right]
$$

A tangential induced velocity decreases with vortex age growth. That is given by growing of a vortex core radius. A vortex core radius size differs in literature within the range of 1 to $7 \%$ of vortex generating airplane span. An initial core size and growth rate was obtained from the literature ${ }^{11,12,13}$. The initial core size value of $1 \%$ of airliner span was used in the extreme formation analysis. The core-growth is described by (2) where an eddy viscosity coefficient $\delta$ is given by (3):

$$
\begin{aligned}
& r_{c}=\sqrt{r_{c 0}^{2}+4 \alpha \delta v t} \\
& \delta=1+a_{1} \operatorname{Re}=1+a_{1} \frac{\Gamma}{v}
\end{aligned}
$$

The value $5 \mathrm{e}-5$ of Squire's parameter $\mathrm{a}_{1}$ was estimated with respect to the literature ${ }^{11}$. The wake vortex model is a steady estimation without turbulence influence and without the first stage of vortex growth. Wake vortex is simulated as pair of vertices. The mutual influence of both vertices causes their vertical decline.

\section{Airliner climb profile}

The climb profile is estimated based on B747 real flight tracking. The source data are collected from a publicly accessible web page monitoring flights with a transponder ${ }^{14}$. Figure 1 presents forward and climb speed history of the airliners. The second part of Figure 1 gives optimal time distance between an airliner and the VWG in a climb regime ${ }^{3}$. The climb of the extreme formation is simulated as a horizontal flight with following modification. Vertical component of induced velocity of the wake vortex is decreased by climb speed $\mathrm{V}_{\mathrm{z}}$ of the airliner.
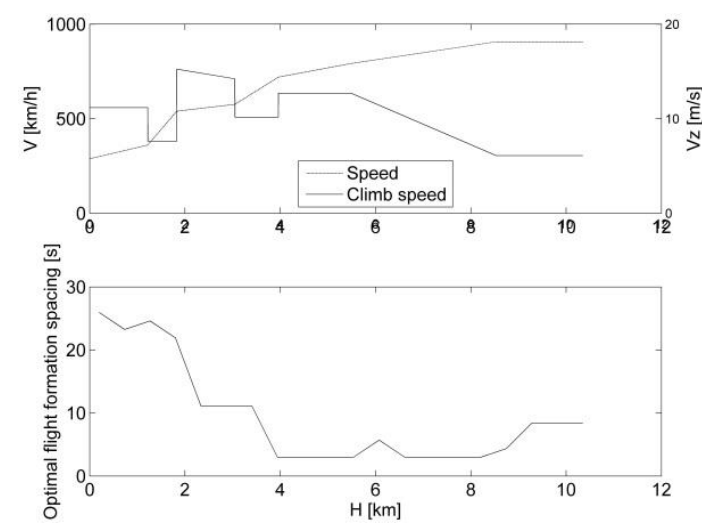

Figure 1. Estimated climb profile of B747 airliner and resultant distance of airliner and WVG

\section{Wake vortex glider drag polar}

The WVG was designed within the previous research with respect to a cruise regime of B747 airliner ${ }^{2}$. Then, weight of the WVG was reduced to reach best performance in a climb regime at the expense of a cruise regime. The main characteristics of the WVG designed for a climb regime are weight of $115 \mathrm{~kg}$, wing area $0.32 \mathrm{~m}^{2}$ and wing span $1.45 \mathrm{~m}$. The sketch of the WVG is shown in Figure 2. 

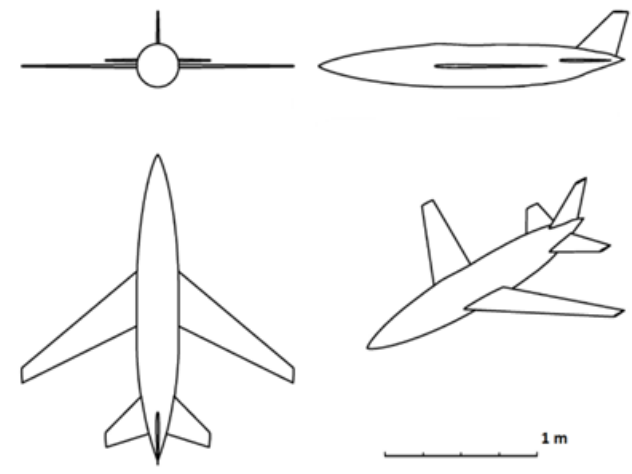

Figure 2. Wake Vortex Glider sketch

Drag polar estimation essentially impacts on results of a gliding flight in a wake vortex. The drag polar was determined by Roskam methodics ${ }^{10,15}$. The polar was estimated with the following analytical form ${ }^{3}(4)$ :

$$
\mathrm{c}_{\mathrm{D}}=\mathrm{c}_{\mathrm{D} 0}+\mathrm{c}_{\mathrm{DL}} \mathrm{c}_{\mathrm{L}}^{2}+\mathrm{c}_{\text {Dtrans }}+\mathrm{c}_{\text {DSaileron }} \delta_{\text {aileron }}
$$

Single parameters reach the following values. The zero drag coefficient $\mathrm{c}_{\mathrm{D} 0}$ is 0.0195 . The drag due to lift coefficient $\mathrm{c}_{\mathrm{DL}}$ is 0.07838 . The drag due to aileron deflection is variable. The coefficient value is taken from ${ }^{16}$ and shown in Figure 3. The aileron is designed along full wing span with $25 \%$ of chord length. Small rudder and elevator deflections required during dynamic soaring are included in the zero drag coefficient. The transonic drag is also defined as a function. The drag coefficient is dependent on Mach number and expressed by graph shown in Figure 3.
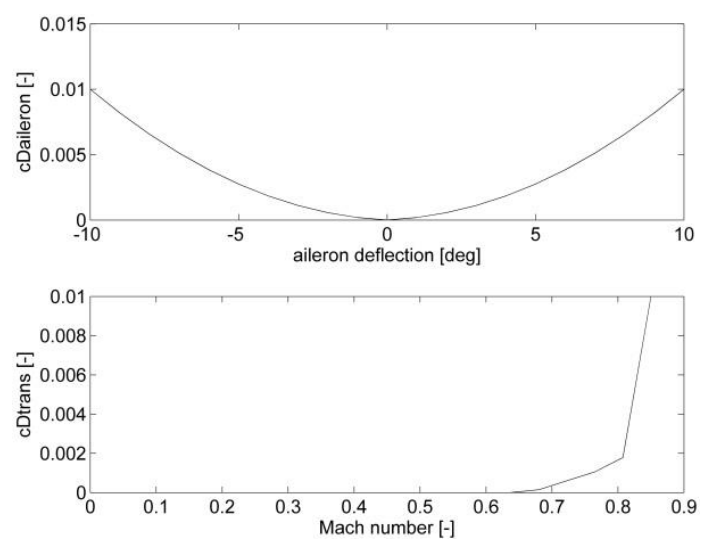

Figure 3. Drag coefficient due to aileron deflections and transonic effect

\section{Dynamic soaring principle}

DS is a specific type of flight where an aircraft or a bird gains energy by manoeuvring in region with wind speed gradient. The principle of energy gain is based on an increase of relative air speed during a flight through the wind speed gradient. An inertial force helps to maintain the ground speed when the soaring object enters stronger headwind region. The relative speed grows in this moment. The aircraft or bird then turns back and gains the relative speed again when crossing the wind gradient in opposite direction and opposite heading. Energy extracted from the speed gradient in such a periodical flight compensates for energy dissipated due to a drag force and allows long time flight without own propulsion.

\section{Methods \\ Equations of motion}

A system of equations of motion for the dynamic soaring simulation is presented in this section. North, East, Down reference frame is used and correspond to $\mathrm{x}, \mathrm{y}$ and $\mathrm{z}$ coordinates. The WVG is simulated as a three degree-of-freedom pointmass model. The following equations of motion which were used by $\mathrm{Zhao}^{5}$ for the dynamic soaring trajectory optimization (5)-(10) are used in the simulation.

$$
\begin{aligned}
& \dot{\mathrm{x}}=V \cos \gamma \cos \psi \\
& \dot{\mathrm{y}}=V \cos \gamma \sin \psi+W_{y} \\
& \dot{\mathrm{z}}=-V \sin \gamma+W_{z} \\
& \dot{\mathrm{V}}=\frac{-D-m g \sin \gamma-m \dot{W}_{y} \cos \gamma \sin \psi}{m}+\frac{+m \dot{W}_{z} \sin \gamma}{m} \\
& \dot{\gamma}=\frac{L \cos \phi-m g \cos \gamma+m \dot{W}_{y} \sin \gamma \sin \psi+m \dot{W}_{z} \cos \gamma}{m V} \\
& \dot{\psi}=\frac{L \sin \phi-m \dot{W}_{y} \cos \psi}{m V \cos \gamma}
\end{aligned}
$$

Where $\psi$ is relative heading angle, $\varphi$ is bank angle and $\gamma$ is relative flight path angle. Induced velocity of a wake vortex is defined by tangential velocity components $\mathrm{W}_{\mathrm{y}}$ and $\mathrm{W}_{\mathrm{z}}$. An axial component of induced speed in the wake vortex model is neglected. A flight along defined trajectory is simulated in Matlab-Simulink. 


\section{Trajectory design}

The trajectory of dynamic soaring flight is designed in a sinusoidal curve lying on a cylindrical section parallel to $\mathrm{x}$ axis. The trajectory shown in Figure 4 is described by the following parameters: amplitude a, period $\mathrm{p}$ and radius $\mathrm{r}$. The other variables are $\mathrm{y}$ and $\mathrm{z}$ position of the cylindrical section axis. The y position of the trajectory is defined by a point with the strongest upwards induced velocity in the wake vortex for given vortex age. The trajectory is anchored to the induced velocity peak during the optimization process.

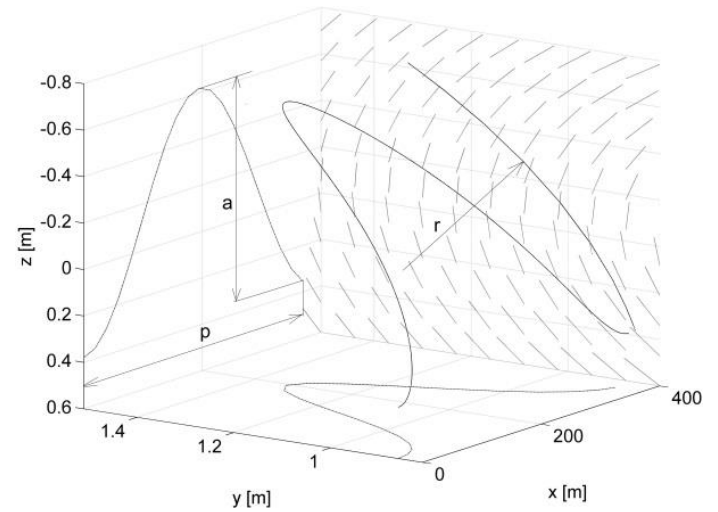

Figure 4. Dynamic soaring trajectory

\section{Optimization method}

Nelder-Mead simplex method ${ }^{17}$ was chosen for optimization of the dynamic soaring trajectory. It is a numerical method used to find the minimum or maximum of an objective function in a multidimensional space. An advantage of the method is the algorithm simplicity with no need to evaluate objective function derivatives. The objective function is defined as WVG energy gain extracted from a wake vortex per one second. The value is evaluated from kinetic and potential energy gained in one flight period divided by the period duration. The optimization variables are defined as follows: period $\mathrm{p}$, radius $r$ and position of the cylindrical section axis $y$. The two remaining are not a subject to the optimization process. The vortex age is taken from the results in a steady flight analysis ${ }^{3}$ depicted in Figure 1. And the amplitude of the dynamic soaring trajectory is defined as a fixed value because of an optimization method convergence. The optimization process is performed for fixed values of the trajectory amplitudes from 0.02 to $1.6 \mathrm{~m}$.

\section{Results}

This section provides results of the trajectory optimization. The trajectory is optimised on a rectangular domain. The amplitude of the dynamic soaring trajectory and the altitude during a climb are independent variables defining the domain. The objective function presents energy gain harvested by the WVG from a wake vortex during $1 \mathrm{~s}$ period.

The Nelder-Mead variant of simplex method converged quickly except a few cases. These cases with slow convergence are connected with the trajectory radius of the cylindrical section growing to very high values. It means that the dynamic soaring trajectory lies near the WVG plane of symmetry. These results are shown in Figure 5 for the amplitudes between 0.1 and $0.6 \mathrm{~m}$.

The trajectory optimization analysis is carried out for the optimal wake vortex age obtained for a steady flight defined in Figure 1. Another vortex age cases are analysed to prove that the optimal dynamic solution do not differ from a steady flight. Figure 5 shows some results solved for vortex ages 3, 4 and $5 \mathrm{~s}$.

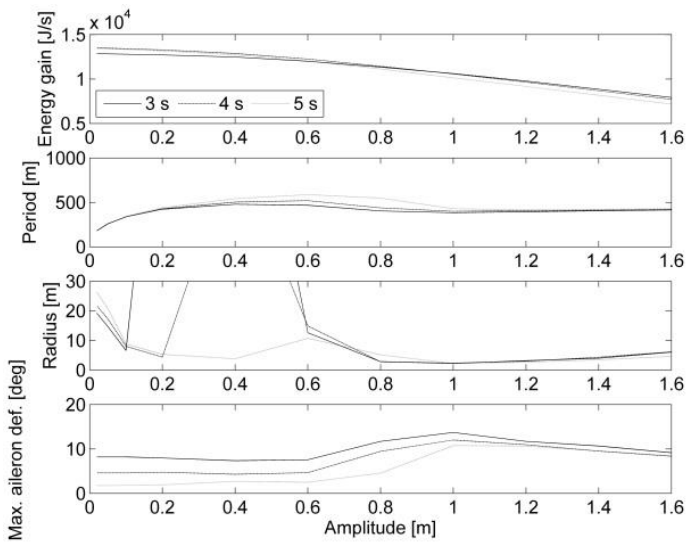

Figure 5. Trajectory optimization results for altitude $5000 \mathrm{~m}$

The first graph in Figure 5 shows very small difference in the energy gain due to the vortex age change. The last graph in Figure 5 shows that required aileron deflections decrease significantly with growing of wake vortex age. It means that controllability of the WVG is getting simpler with growing vortex age. 


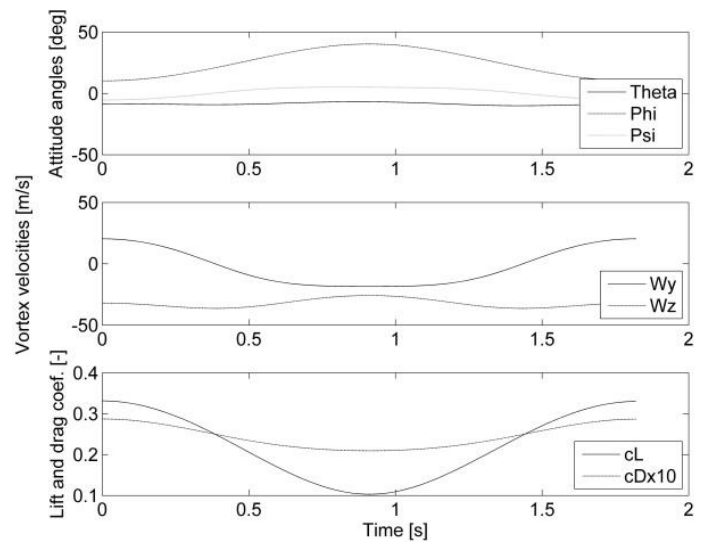

Figure 6. Variables history of example trajectory for altitude $5000 \mathrm{~m}$ and amplitude $1 \mathrm{~m}$

Time history of some variables is shown in Figure 6. The results are obtained for the dynamic soaring amplitude $1 \mathrm{~m}$ at the altitude of $5000 \mathrm{~m}$. All variables are plotted for one period of the sinusoidal trajectory. The beginning of the trajectory lies on the lowest point of the curve as shown in Figure 4. The first graph of Figure 6 gives history of the attitude angles $\psi, \varphi$ and $\gamma$. The curves are plotted in the interval of one period of the dynamic soaring motion. The middle graph of Figure 6 shows the induced velocity components along the optimal trajectory coordination. Vertical induced velocity $\mathrm{W}_{\mathrm{z}}$ of the wake vortex is already reduced by the airliner climb speed because of a climb regime simulation. The last graph depicts lift and drag coefficients history. Lift coefficient of the WVG varies from 0.33 to 0.1 . Lift and drag coefficients correspond to a gliding ratio varying from 11.5 in the lowest point to 5 in peak of the trajectory.

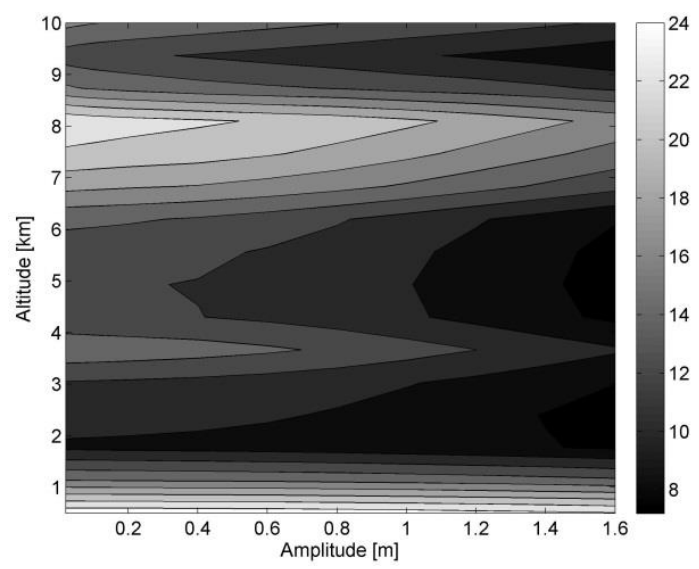

Figure 7. Energy gain per second $[\mathrm{kJ} / \mathrm{s}]$ objective function results for different altitudes and trajectory amplitudes
Figure 7 provides resultant values of the objective function. The objective function presents the energy gain in $\mathrm{kJ}$ per one second extracted from a wake vortex by the WVG during dynamic soaring. The results are plotted in the flight altitude and the DS amplitude domain.

Figure 8 presents maximal required aileron deflections during the dynamic soaring flight. The figure is plotted in the flight altitude and the DS amplitude domain. The values in degrees correspond to a mean aileron deflection for differentiated left and right ailerons.

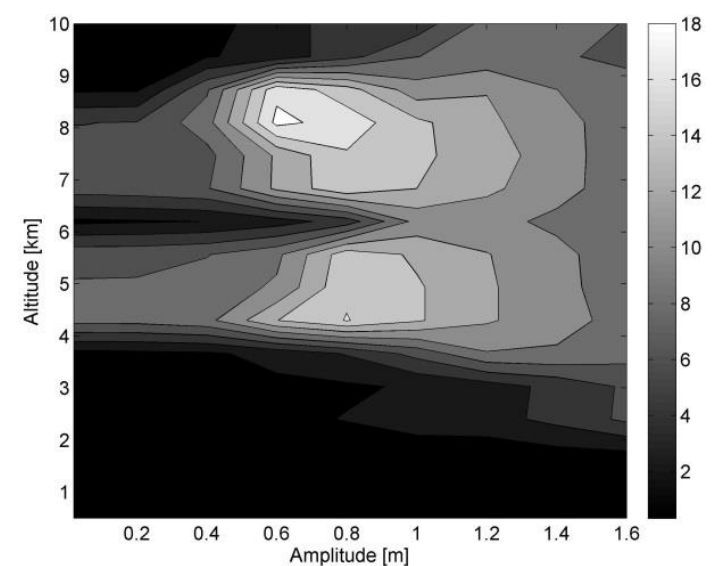

Figure 8. Maximum aileron deflections $\left[{ }^{\circ}\right]$ in the optimal trajectory flight

\section{Discussion}

The results of the trajectory optimization process are discussed in this section. DS energy gain was proved in spite of the energy decrease in Figures 5. and 7. The proof is radius $r$. It would converge to infinity value in a case that the principle of DS is wrong. The radius causes that the trajectory deviates from a region with the strongest upwards induced velocity in a wake vortex. This fact confirms that DS allows energy extraction from a wake vortex. On the other side, the WVG extracts less energy from a wake vortex during a DS flight in comparison to a steady flight. The energy gain decreases with growing amplitude of the DS trajectory. The objective function represents energy gain per second by the WVG from a wake vortex. The energy decreases in all altitudes with growing amplitude of the DS trajectory. The DS flight is not the optimal flight in a wake vortex but the analysis result shows that DS is theoretically possible. The energy extraction is not the only reason for DS. The DS flight could be also required because of searching for the optimal position for the WVG 
in a wake vortex. The next result is a controllability validation during the DS flight.

Figure 8 provides required aileron deflection for the DS flight. The results are plotted over the whole optimization domain. There are two peaks around altitudes 4000 and $8000 \mathrm{~m}$. The optimal trajectory with the amplitudes around 0.6 and $0.8 \mathrm{~m}$ requires high aileron deflections at these altitudes. The altitude around 4000 and $5000 \mathrm{~m}$ is a critical phase during the whole climb from controllability point of view. The surplus energy gain is much smaller than at altitudes around $8000 \mathrm{~m}$. The energy gain can be easily converted to better controllability by increasing of a distance between an airliner and the WVG.

The DS analysis does not reveal energy gain benefit in comparison to a steady flight in a wake vortex. Energy gain is not the only benefit from DS on the other side. Navigation to a peak of upwards induced velocity in a wake vortex is an important task in a formation flight ${ }^{18}$. The DS flight pattern allows scan the wake vortex induced velocities continually. This is a necessary condition of a wake vortex flight in turbulent atmosphere. The decrease of energy gain in the DS flight is acceptable value paid for navigation improvement in a wake vortex.

\section{Conclusions}

The DS analysis extends results already published for a steady flight in a wake vortex. Theoretical background is presented in the first sections. Wake vortex characteristics, an airliner climb profile and the WVG drag polar are taken from the previous research. The essential part of the paper describes the trajectory optimization of DS motion in a wake vortex by Nelder-Mead variant of Simplex method.

The DS principle works in a wake vortex but the benefit decreases with rising amplitude of the trajectory. The results do not validate hypothesis about energy gain benefit in comparison to a steady flight in a wake vortex. The other important result of the analysis deals with controllability of the WVG. The glider can be controlled with suggested control surfaces during a DS flight. But it needs to avoid a wake vortex axis proximity where induced velocity gradient is too strong and the glider cannot compensate roll moment.

The concept of the wake vortex flight seems to be feasible but some questions need to be solved. How the UAV take off and approach an airliner wake vortex? What is maximal turbulence in atmosphere for the extreme flight formation? How to navigate the UAV into the best position in a wake vortex? Can be the UAV equipped by any electrical turbine to generate energy in a wake vortex and produce thrust when get out of the wake vortex? These questions open possibilities for future research in the field of the airliner-UAV formation flight.

\section{Acknowledgment}

The work leading to these results was supported by Czech Science Foundation in the frame of GP14-16370P project "Wake vortex gliding as a new propulsion concept for Unmanned Aerial Vehicles".

\section{References}

[1] Zikmund P, Popela R. Wake vortex gliding as a new propulsion concept for UAVs. Czech Aerospace Proceedings 2013; 2013(2): 14-16.

[2] Zikmund P. Wake vortex gliding. 6th EUCASS Conference, Krakow 2015.

[3] Zikmund P, Popela R. Airliner-UAV Flight Formation in Climb Regime. World Academy of Science, Engineering and Technology, International Science Index 106, International Journal of Mechanical, Aerospace, Industrial, Mechatronic and Manufacturing Engineering 2015; 9(10): 1661 - 1666.

[4] Lange KO. Soaring meteorology. In The Art of Gliding and Soaring. London: Barringer LB (ed.). Pitman Publishing Corporation; 1942.

[5] Zhao YJ. Optimal patterns of glider dynamic soaring. Optimal control applications and methods 2004; 25(2): 67-89. (DOI: 10.1002/oca.739)

[6] Denny M. Dynamic soaring: aerodynamics for albatrosses. European Journal of Physics 2009; 30(1): 75. (DOI:10.1088/0143-0807/30/1/008)

[7] Bower GC. Boundary layer dynamic soaring for autonomous aircraft: Design and validation. Doctoral dissertation, Stanford University; 2012.

[8] Grenestedt J, Montella C, Spletzer J. Dynamic soaring in hurricanes. Proceedings of the ICUAS 2012.

[9] Grenestedt J, Spletzer JR. Towards perpetual flight of a gliding unmanned aerial vehicle in the jet stream. Decision 
and Control (CDC), 2010 49th IEEE

Conference: 6343-6349.

[10] Kóňa M. Aerodynamic design of transonic glider. Master thesis, Brno University of Technology; 2015.

[11] Bhagwat MJ, Leishman JG. Generalized viscous vortex model for application to free-vortex wake and aeroacoustics calculations. Annual Forum ProceedingsAmerican Helicopter Society 2002;20422057.

[12] Ahmad NN, Proctor FH, Duparcmeur FML, Jacob D. Review of idealized aircraft wake vortex models. AIAA 2014; Available from:

http://ntrs.nasa.gov/search.jsp?R=201400 03974

[13] Delisi PD, Greene GC, Robins RE, Vicroy DC, Wang FY. Aircraft wake vortex core size measurements. 21st Applied Aerodynamics Conference, Orlando, Florida 2003; 1-9.

[14] Flightradar24.www.flightradar24.com (accessed June 2015).

[15] Roskam J. Airplane Design VI: Preliminary calculation of aerodynamic, thrust and power characteristics. Kansas; 1990.

[16] Lockwood VE, Fikes JE. Control characteristics at transonic speeds of a linked flap and spoiler on a tapered $45^{\circ}$ sweptback wing of aspect ratio 3. NACA RM L52D25; 1952.

[17] Nelder JA, Mead R. A simplex method for function minimization. Computer Journal 1965; 7: 308-313.

[18] Chichka DF, Speyer JL, Fanti C, Park CG. Peak-seeking control for drag reduction in formation flight. Journal of guidance, control, and dynamics 2006; 29(5): 1221-1230. 